\title{
The Effect of Handedness on Supplementary Motor Area Activation during Complex Motor Tasks
}

\author{
Amauri Dalla-Corte', Carlos M. M. das Neves², Maurício Anés ${ }^{2}$, Mirna W. Portuguez ${ }^{1,3}$, \\ Jaderson C. Dacosta ${ }^{1,3}$ \\ ${ }^{1}$ Post-Graduation Program in Medical Clinics and Health Sciences, School of Medicine, Pontifical Catholic \\ University, Porto Alegre, Brazil \\ ${ }^{2}$ Image Diagnostic Center of the São Lucas Hospital, Pontifical Catholic University,Porto Alegre, Brazil \\ ${ }^{3}$ Brain Institute (InsCer), Pontifical Catholic University, Porto Alegre, Brazil \\ Email: dalacorte@gmail.com
}

Received 3 August 2015; accepted 26 September 2015; published 29 September 2015

Copyright (C) 2015 by authors and Scientific Research Publishing Inc.

This work is licensed under the Creative Commons Attribution International License (CC BY).

http://creativecommons.org/licenses/by/4.0/

(c) () Open Access

\section{Abstract}

Functional magnetic resonance imaging (fMRI) was used to assess the activity of supplementary motor area (SMA) in six right-handed and six left-handed healthy volunteers. Two manual tasks (self-initiated previously practiced and unpredictable visually guided) were used. Quantitative analysis of hemispheric and bilateral SMA activation was described as mean \pm standard deviation of hot spots/total spots. The two tasks induced bilateral SMA activation. The laterality of SMA activation was affected by manual dominance. Left SMA was significantly more activated in rightand left-handers while performing the motor tasks with the right hand. Right SMA was more activated in the left-handers when the left hand was used. Task complexity was the most important factor influencing the degree of SMA activation.

\section{Keywords}

Functional Magnetic Resonance Imaging, Motor Control, Supplementary Motor Area, Laterality, Handedness

\section{Introduction}

Recent development of neuroimaging techniques opened new possibilities to investigate the relation between handedness and brain functional organization, notably with respect to hemispheric specialization, which might 
cause the neural substrate of hand dominance or skill to differ between the right- and left-handed subjects. Approximately $90 \%$ of people are right-handed and in the majority the left hemisphere is dominant for manual abilities [1]. Also there is strong evidence for a handedness-associated anatomical hemispheric asymmetry. However, there is no agreement as to whether these activations are characteristic of the non-dominant hand or both hands. Moreover, previous studies indicate that task complexity has an important impact on differences in the motor activation pattern associated with dominant and non-dominant movements [2]-[4].

Several studies have sought to demonstrate the role of SMA in special motor functions, such as initiation and self-regulation [5]-[8], in addition to its important role in the internal preparation and control of complex and sequential movement [9]. In fact, the SMA is not supplementary, but it is motor in the sense that it is programming areas for motor subroutines. Other authors suggest that the SMA can also control the movement independently of the primary motor cortex (M1), in addition to participating in the coordination on both sides of the body [10] [11]. Anatomical and physiological data available suggest that the SMA could be the site where external inputs and commands join with the internal needs to facilitate the programming of a strategy of voluntary movement [12]. The stimulation threshold of the SMA requires electrical stimuli with intensity and duration far greater than those produced by stimulation of M1, and the responses extracted from SMA are ipsilateral or often bilateral and may also be simultaneous [13].

Since the results of activation studies have not been entirely consistent, we wanted to look beyond them and verify the hypothesis of a hemispheric lateralization and asymmetry in SMA. To that end, we used two different complex sequential movements: a self-initiated and an externally triggered, and compared the SMA activation patterns obtained in accordance with the handedness.

\section{Materials and Methods}

\subsection{Sample}

Twelve healthy volunteers aged 25 - 40 (average $30.5 \pm 4.1$ ) years and with third level education were recruited through a newspaper announcement (Table 1). The subjects were classified as right-handed (6) or left-handed (6) through the test for Manual Dominance (Handedness Inventory) [14]. A questionnaire designed to exclude any neurological and/or psychiatric disorder that could alter the performance was first completed by each volunteer. In all of them, the neurological examination was normal and the MRI excluded any type of malformation or expansive lesion of the central nervous system. Additional exclusion criteria included hearing impairment or contraindications for MRI scanning (e.g., metal implants, pacemakers, pregnancy and claustrophobia). The study was approved by the Ethics Committee of the Pontifical Catholic University of Rio Grande do Sul (PUCRS), Porto Alegre, Brazil, and was performed with the collaboration of the Image Diagnostic Center of the São Lucas Hospital. All the subjects gave their written informed consent.

\begin{tabular}{cccc}
\hline \multicolumn{3}{l}{ Table 1. Characteristics of the study sample. } \\
\hline Subjects & Gender & Age & Handedness \\
\hline AB & F & 25 & Left \\
AA & F & 27 & Left \\
AD & M & 31 & Right \\
AB & F & 30 & Right \\
CT & F & 29 & Left \\
CMMN & M & 40 & Right \\
DB & M & 26 & Right \\
GEP & M & 32 & Left \\
JHNP & M & 34 & Left \\
LFA & M & 28 & Left \\
RMP & F & 34 & Right \\
RRD & F & 30 & Right \\
\hline
\end{tabular}




\subsection{Image Acquisition}

Experiments were performed on a 1.5 Tesla Siemens Magnetom Vision System by using a standard head coil. In order to avoid movement artifacts, the volunteers' heads were aligned with two steadying arms that formed part of the coil and all the participants were instructed not to move their heads. The image acquisition parameters were previously described [15]. A motion-correction factor was also applied. In each task run, a series of 108 measurements was acquired, each one with 17 images and a time interval of 3-s between each measurement. A total of five series of 12 measurements with the subject at rest, interspersed by four series of 12 measurements during finger-tapping were obtained. The entire experiment consisted of two experimental runs with each hand. The images were processed and the functional maps obtained by use of the software program Stimulate 5.8 from the University of Minnesota, Minneapolis, USA [16] installed on a Silicon Graphics workstation. The data analysis was performed by comparing the EPI images obtained during the rest (control) periods and the task periods using Student's $t$-test. Afterwards, the data from each experiment were normalized for the Talairach coordinates, and the individual maps were compared voxel by voxel after sagittal reconstruction in 3D (spatial) and SMA anatomical delineation in accordance with parameters that will be described below. Each volunteer generated the following data: proportion of the number of hot (activation) spots as against the total number of spots included in the SMA (bilateral, left, and right) in both tasks (A and B)—-these were inserted into a Microsoft Excel program chart and later submitted to statistical analysis.

\subsection{Activation Paradigms}

Following an order from the examiner to start the tasks, the subject should alternately touch the thumb with the finger tips on the hand indicated until told to stop. In case of error, the sequence should be restarted. The sequences of finger movements used were: A) 16 finger-to-thumb opposition movements with fingers 2-5: 2-2-34-4-4-5-5-5-5-4-4-4-3-2-2; B) 12 finger-to-thumb opposition movements with fingers 2-5: 5-4-3-5-4-2-5-3-24-3-2 (omitting one subsequent finger each time). The self-initiated sequence (A) described by Roland et al. [8] was shown 20 minutes before the exam, so the subjects could train to perform a quick sequence. The externally triggered (B) [17] had an unpredictable nature. The subjects viewed the task pictures projected onto a screen through a mirror attached to the head coil in the scanner (Figure 1). Images prepared in Microsoft Power Point program, consisted of hands, right and left, with signs of the fingers that progressively should be touched, alternating every second (paced at $1 \mathrm{Hertz}$ ) and interrupted by the inscription STOP at the end. The 36-s rest periods were followed successively by 36-s task periods, once with the right hand, once with the left hand.

\subsection{Delineation of the SMA in FMRI}

The SMA was delimited from the Talairach space that is defined in a sagittal section by the intersection of the

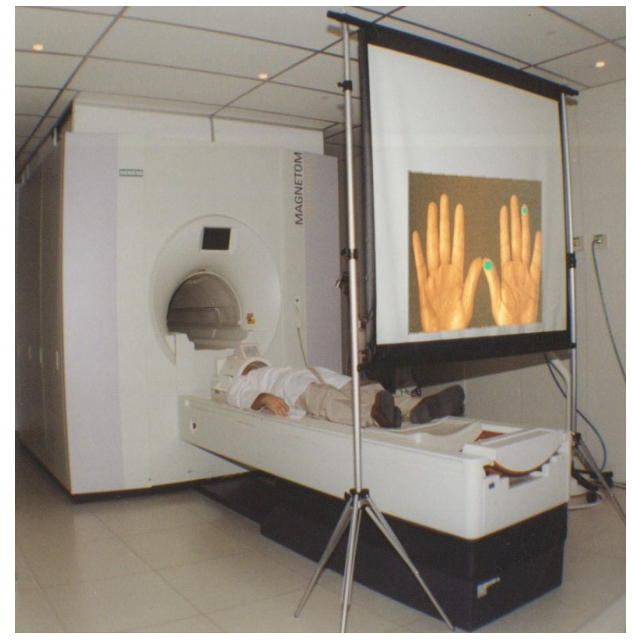

Figure 1. Siemens magneton vision system during the unpredictable projected manual task (task B). 
bicommissural line (anterior commissure-posterior commissure) and a perpendicular line crossing the anterior commissure (VCA) at the midline [18]. The VCA line is the anatomical anterior limit, separating the pre-SMA (rostral to the VCA line) from the SMA proper (caudal to the VCA line). Another line, also perpendicular and which crosses the posterior commissure (VCP) at the midline coincides with the medial precentral sulcus, which properly defines the posterior limit of the SMA and separates the Brodmann area 6 from area 4 [19] [20]. SMA is also limited to the cortex on the medial wall of the hemisphere, extending from the top of the brain to the depth of the cingulated sulcus, including the dorsal bank of the cingulate sulcus [21] [22].

\subsection{Statistical Analysis}

The quantitative data on the hemispheric and bilateral activations of the SMA (hot spots/total spots) were described by using the average and standard deviation. For the categorical data, we used frequencies and percentages. The comparisons between the readings were made with Student's $t$-test for independent or paired samples as the situation required. Event comparisons were made using the Binomial test and, when necessary, Fisher's exact test [23]. In the dispersion graphs, we calculated the residuals of linear regression line to obtain estimates of the hemispherical activations [24] and established the convention that the right side is positive and the left is negative. The equation used for obtaining the residuals was: res $=-(y-x)$. As an aid to the evaluation of the magnitude of the differences obtained in the comparisons, we used the statistic of the effect size (ES) and Hopkins's criteria to interpret its magnitude [25].

\section{Results}

\subsection{Activation of the SMA during Two Sequential Voluntary Movements}

Between the self-initiated (task A) and unpredictable projected (task B) manual tasks, task A performed with the right hand showed a greater degree of activation in the bilateral SMA (18.6\%) (Figure 2(a)), where the left SMA was more activated (21.8\%) (Figure 2(b)) than the right (16.8\%) (Figure 2(c)). Comparing the mean activation values obtained in tasks A and B performed with the same hand, a systematically greater activation with task A (6 of 6 events; $\mathrm{p}=0.016$ ) was observed, with a mean difference of 3 percentage points (Figure 2). Among the 6 comparisons carried out between tasks A and B, the highest activation occurred in the left SMA with the movement of the right hand, where the difference was as high as 5.6 percentage points $(21.8$ - 16.2) (Figure 2(b)).

\subsection{Comparison between Right- and Left-Handers}

Task A performed with the right hand produced the highest mean activation value in the bilateral SMA either in the right-handed (20.5\%) or left-handed group (16.7\%) (Figure 3(a)). In this task, the left SMA was proportionally more activated in both groups (24.4\% in the right-handed and $19.3 \%$ in the left-handed) compared to the right SMA (18.5\% in the right-handed and 15.0\% in the left-handed) (Figure 3(b), Figure 3(c)) as shown in the example of a volunteer in Figure 4. In task A with the left hand, we found a significant difference in favor of right-handers (Figure 3(a)), mainly when considering only the left SMA activation $(E S=0.69)$ (Figure 3(b)). The use of the left hand in the left-handers produced the lowest mean activation values in the bilateral SMA and there was practically no difference between tasks A (12.6\%) and B (12.0\%) (Figure 3(a)), as it also happened in the left SMA (Figure 3(b)). However, in the right-handers, when using the dominant hand, the difference in activation between tasks A (20.5\%) and B (14.0\%) was significant in favor of task A (ES = 0.62) (Figure 3(a)), and a little higher in the right SMA (Figure 3(c)). Task B carried out with the right hand caused lower activation in the right-handed group (14.0\%) (Figure 3(a)), mainly in the right SMA (11.0\%) (Figure 3(c)). Task B executed with the left hander caused lower activation in the left-handed group (12.0\%) (Figure 3(a)), especially in the left SMA (10.4\%) (Figure 3(b)). Left SMA activation with tasks A and B was always higher in the right-handers, except in task B performed with the right hand, in which the mean activation value was equivalent between left-handers (16.3\%) and right-handers (16.1\%). Also related to the left SMA activation, we observed among the left-handers a significant difference in favor of the use of the right hand in both task A (ES $=0.83$ ) and in task $\mathrm{B}(\mathrm{ES}=0.88)$. In the left-handed group, the greatest activation in the right SMA was obtained with task B carried out with the right hand (15.8\%) as shown in the example of a volunteer in Figure 5. In this task, there was the greatest but non-significant activation difference between right- and left-handers in the right SMA. 


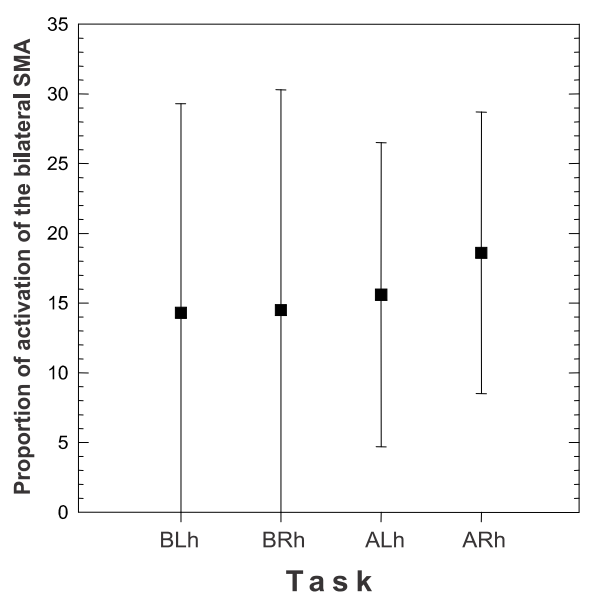

(a)

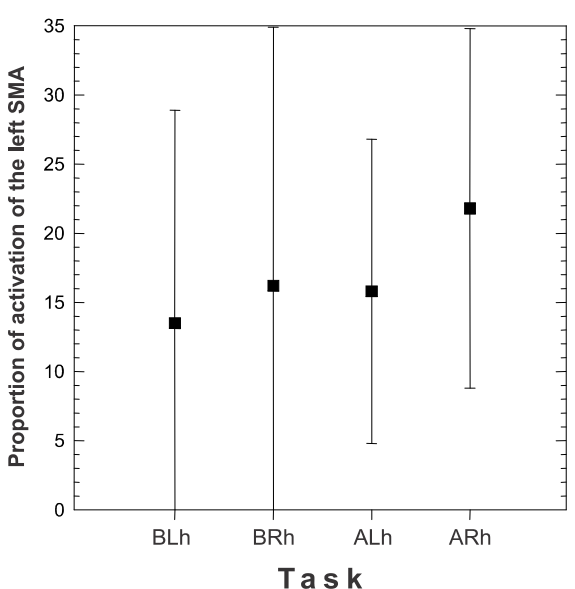

(b)

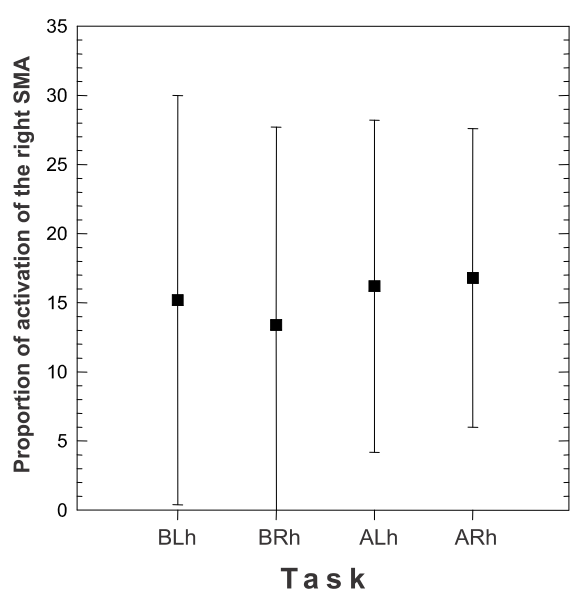

(c)

Figure 2. Bar chart of error presenting average \pm standard deviation for the proportion of the bilateral (a), left (b) and right (c) SMA activation in all volunteers (right- and left-handers) according to the task and the hand used. $\mathrm{BLh}=$ task $\mathrm{B}$, left-hand; $\mathrm{BRh}=$ task $\mathrm{B}$, right hand; $\mathrm{ALh}=$ task $\mathrm{A}$, left hand; $\mathrm{ARh}=$ task $\mathrm{A}$, right hand.

A greater number of activations with ipsilateral predominance was demonstrated in the right SMA with tasks A and B in the group of left-handers and only with the task A in the group of right-handers (Figure 3(c)).

In the left-handers, tasks $A$ and $B$ performed with the left hand produced a greater activation in the right SMA (res $_{\mathrm{hh}}$ task $\mathrm{A}=2.7$; res $_{\mathrm{hh}}$ task $\left.\mathrm{B}=4.4\right)$. In the same tasks the right-handers had a tendency toward neutrality with a small excess toward the left side (res ${ }_{\mathrm{rh}}$ task $\mathrm{A}=-1.9$; res $_{\mathrm{rh}}$ task $\mathrm{B}=-1.1$ ) (Figure 6(a) and Figure 6(c)). In this situation, there was a significant difference in favor of the left-handers both in task B $(E S=1.12)$ and task A (ES = 0.89). In tasks A and B performed with the right hand, there was greater activation in the left SMA by right-handers ( res $_{\mathrm{rh}}$ task $\mathrm{A}=-5.9$; res $_{\mathrm{rh}}$ task $\mathrm{B}=-5.1$ ). In task $\mathrm{A}$ with the right hand, the left-handers also showed greater activation in the left SMA, but had a tendency toward neutrality in task B (res lh $_{\mathrm{h}}$ task $\mathrm{A}=-4.3$; res $_{\text {lh }}$ task B $=-0.4$ ) (Figure 6(b) and Figure 6(d)). Thus, in task B with the right hand, there was a significant difference in favor of the right-handers ( $\mathrm{ES}=0.6)$.

In the majority of the tasks (6 of 8 events; $\mathrm{p}=0.109$ ) there was a predominance of activation in the left SMA (Figures 6(a)-(d)). Task B did not determine any activation in the SMA in two volunteers with left handedness regardless of the hand used. Task $B$ executed with the right hand also did not demonstrate activation in one right-handed volunteer (Figure 7). However, task A induced activation in the SMA in all twelve subjects tested.

\section{Discussion}

The medial wall of the frontal lobes appears to be involved to a similar degree both in internal and external tim- 


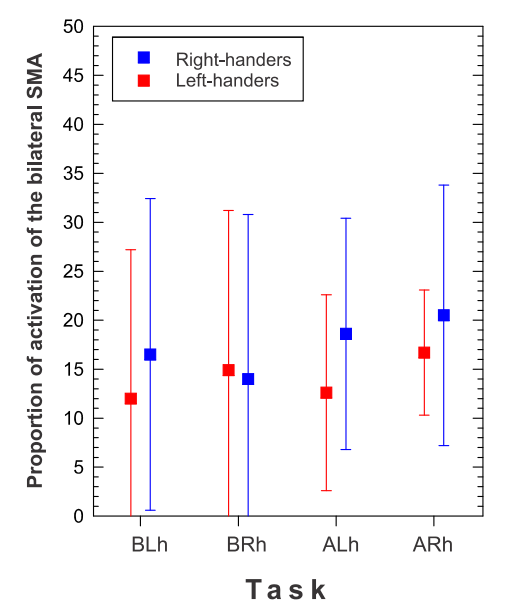

(a)

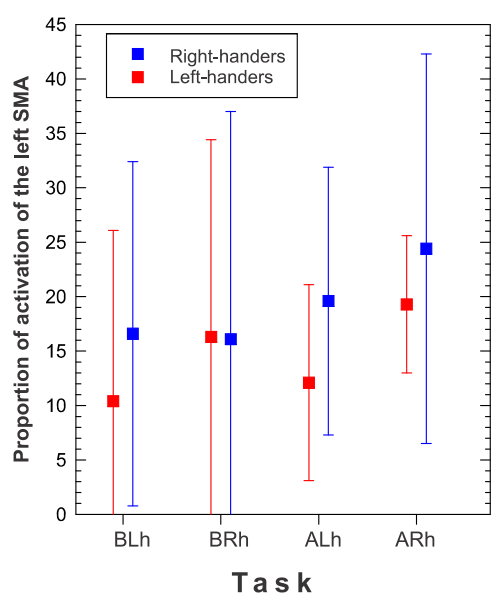

(b)

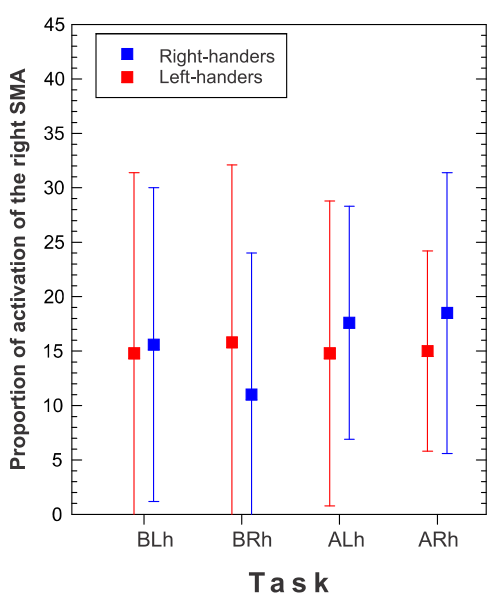

(c)

Figure 3. Bar chart of error presenting average \pm standard deviation for the proportion of the bilateral (a), left (b) and right (c) SMA activation in right- and left-handers according to the task and the hand used. BLh = task $\mathrm{B}$, left-hand; $\mathrm{BRh}=$ task $\mathrm{B}$, right hand; $\mathrm{ALh}=$ task $\mathrm{A}$, left hand; $\mathrm{ARh}=$ task $\mathrm{A}$, right hand.

ing mechanisms [26] [27], supporting the temporal organization of multiple movements [28] [29]. Many of the paradigms that have been tested demonstrate relationship with the increase of the fMRI signal in the SMA, amongst which are the complexity of the movement, the self-initiation or the external signalization, the unpredictability of the task and its time of initiation, the bimanual coordination and the motor learning [22] [30]-[37].

In this study, there was a significantly greater SMA activation with the self-initiated task, which is more complex than the externally triggered task. Using the same complex sequence that we applied, other authors demonstrated that the activity in the SMA depends on the complexity of the unilateral manual movement [3] [4].

fMRI studies using visually guided motor task demonstrated increased activation of multiple secondary motor areas when the movement sequence is unknown without respective signal change in the M1 [22] [35]. Thus, with the aim of increase SMA activation, the complexity and the unpredictability were used separately in each task of this study. However, despite the unpredictability of the visually projected manual task intending to exclude the access to already established motor programs, we demonstrated a predominance of SMA activation with the self-initiated motor task. This supports the idea that the task with its respective degree of complexity is the principal determinant of SMA activation. Another hypothesis to be considered is that the SMA appears to be involved in the preparation of movement sequences through memory in the absence of visual signals as demonstrated by the cortical neuronal recordings in monkeys [6] [28]. This is in agreement with various authors who indicate a crucial role of the SMA in learned and internally generated voluntary motor behavior, especially in 

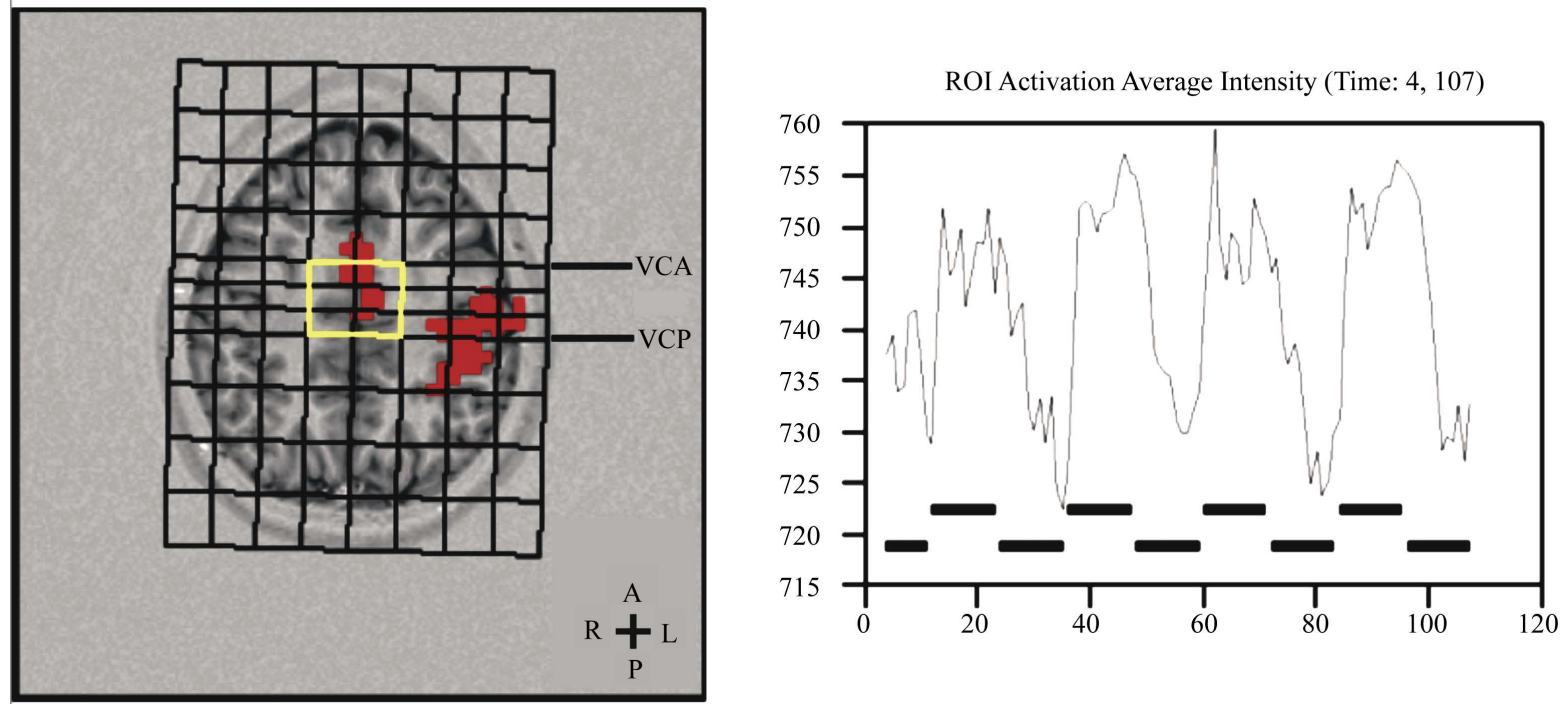

Figure 4. Image of functional activation (red hot spots) in the bilateral SMA of volunteer AB, left-hander, while executing the task A with the right hand, and the respective graph of activation during the rest and task periods.

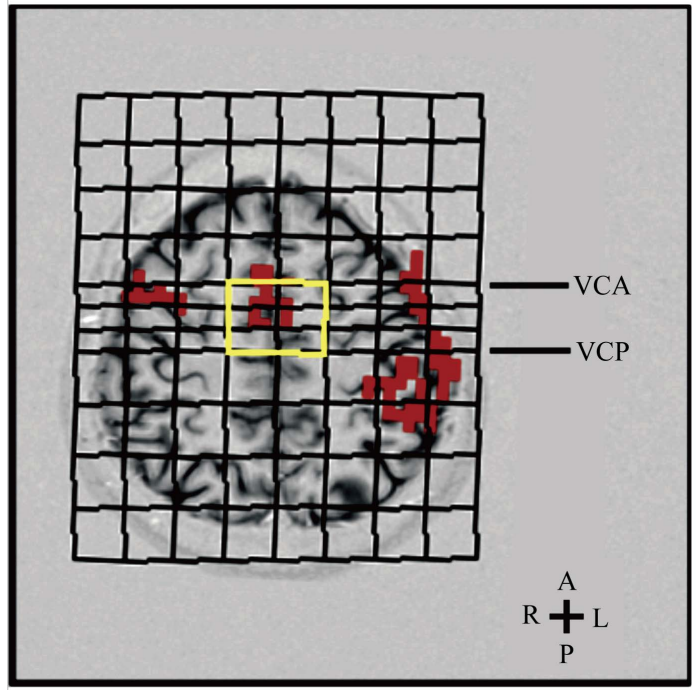

Figure 5. Image of functional activation (red hot spots) in the right SMA of volunteer JHNP, left-hander, while executing the task $\mathrm{B}$ with the right hand.

the preparation and initiation of complex tasks [2] [38] [39].

The fact that both SMA are activated during sequential manual movements as demonstrated by this study could indicate that the elaboration of motor subroutines is bilateral. Studies using PET and fMRI, including right- and left-handed volunteers, demonstrated bilateral SMA activation related to unilateral movement, especially when it was complex [2] [8] [40]. Anatomical and surgical data support the idea that the SMA consists of a bilaterally organized system and probably contributes to bimanual coordination [13] [41]. This can explain our findings of bilateral SMA activation, however, with a predominance of activation in the SMA contralateral to the hand used in both manual tasks.

On examining the influence of handedness on SMA activation, our findings suggest that the complexity of the manual task executed with the dominant hand has a greater SMA activation effect in right than left-handers. Also there is no literature consensus about the asymmetry of contralateral activation in the sensorimotor cortex between right- and left-handed people [42] [43]. Kim et al. studied right- and left-handed subjects with fMRI 


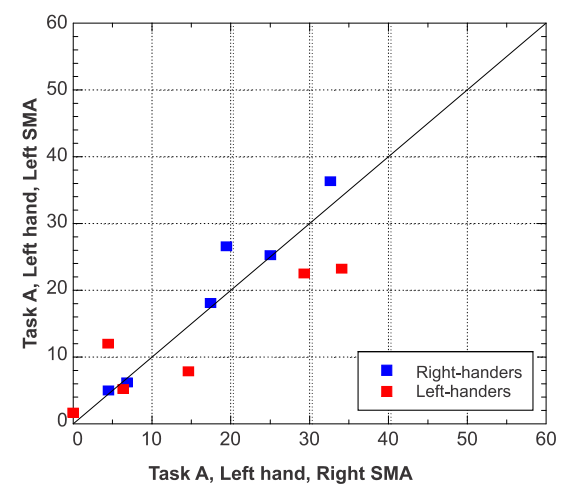

(a)

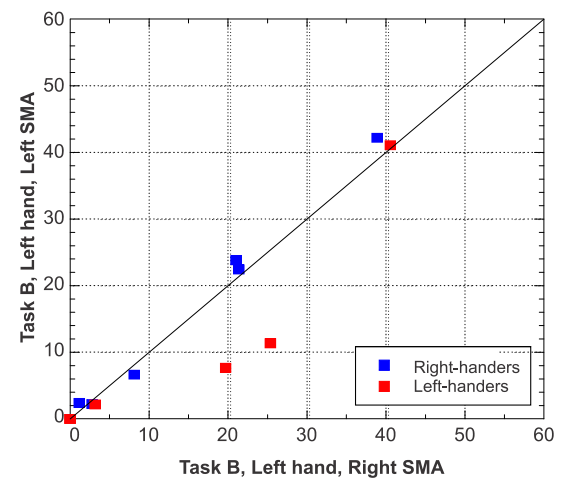

(c)

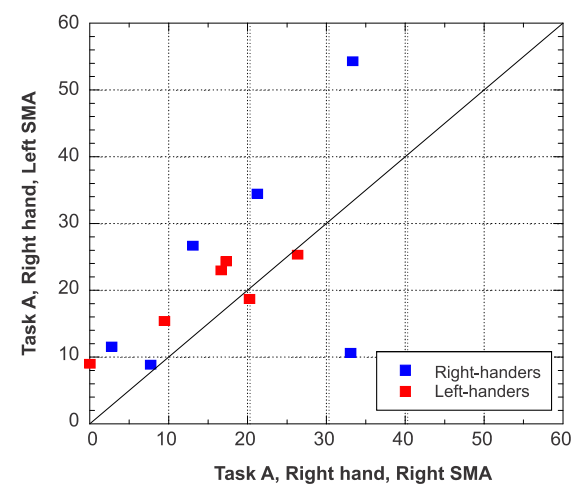

(b)

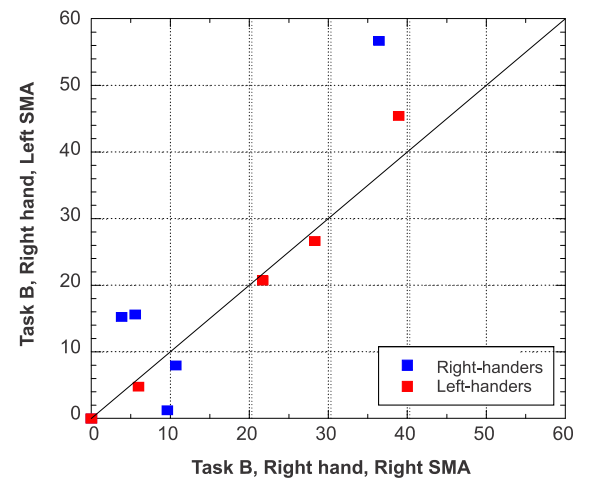

(d)

Figure 6. Dispersion graphs representing the distances distribution of the average activation line between the right and left SMA for right- and left-handers according to the task and the hand used.

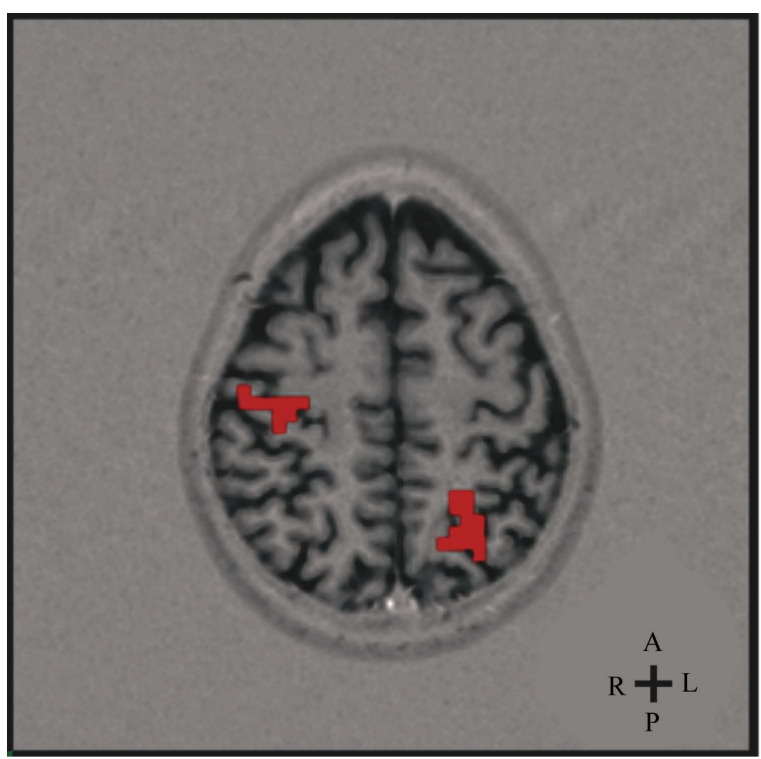

ROI Activation Average Intensity (Time: 4, 107)

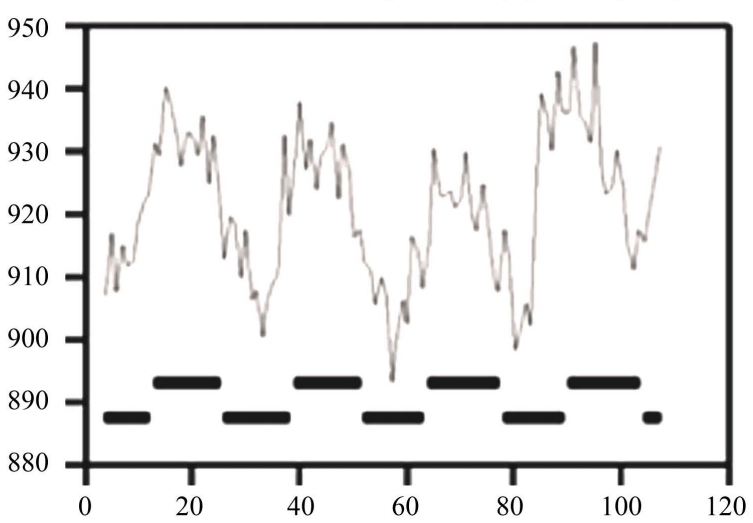

Figure 7. Image of volunteer RRD, right-hander, while executing the task B with the right hand, without functional activation (red hot spots) in the SMA, and the respective graph of activation during the rest and task periods.

and suggested that the reason for this asymmetry found especially in right-handers lies in the difference in the complexity of manual movement, which is difficult for right-handers when performed with the left hand [1]. An established result is that the inter-manual difference in hand skill is most often reduced in left-handers [44], 
while right-handers exhibit a stronger degree of hand preference at handedness inventories [45]. These differences in neuronal effort substantially contribute to handedness-specific activation patterns during manual motor tasks [46].

In the first publication addressing SMA and handedness, Dassonville et al. described a hemispheric dominance of the left human SMA in right- and left-handers [22], which was in accordance with the data of the present study. The explanation may lie in the fact that there are monosynaptic motoneuronal connections projected directly from the SMA (94\% contralateral and 6\% ipsilateral) [13], that special movement conditions determine a greater activation in the left SMA, or in fact that the left hemisphere is dominant for movement control.

The right SMA activation, contrary to what one might expect, was not always higher in left-handers, which again demonstrated that the difficulty of the task seemed to have a greater impact on the degree of SMA activation in the right-handed group. Mattay et al. also found greater ipsilateral SMA activation during a more complex task with the dominant hand in right-handed volunteers [47]. According to Fried, the right SMA may be part of the right cerebral system dominant for attentional and intentional mechanisms directed at the body and the external space in which motor activity takes place [48]. Thus, the right SMA is more involved in bilateral motor control, whereas the left SMA is involved primarily in control of the contralateral (right) side of the body, and consequently the left side of the body may have less supplementary motor representation than the right side.

Other authors also reported that ipsilateral activations are more pronounced during left hand movements executed by a right-hander and depend on the complexity of the task [2] [3] [49]-[51]. Entirely in line with the results reported here, Verstynen et al. showed that hemispheric asymmetries observed in dorsal premotor cortices during ipsilateral movements of left-handed people tended to decrease for more complex movements [52]. These and other authors also observed a marked tendency towards left hemispheric involvement in complex motor control for both right- and left-handed subjects hemisphere activation, instead of the expected contralateral dominance and regardless of which side the task was performed on, which was consistent with this study and suggested that this effect reflected rather hemispheric specialization than handedness [40] [52].

In some situations no SMA activation was observed with the unpredictable visually guided task, indicating in these cases a probably individual variation in which the SMA participation was directed towards more selected aspects of movement. According to Scholz et al., whereas various motor tasks invariably produce motor cortical activation, basal ganglia and SMA occasionally do not demonstrate activation [40]. There is also evidence that the individual differences in activation are related with the underlying brain structure more specifically with the gray matter [45].

Because of the possibility of motor learning and lower SMA activation in one right-handed volunteer who had explained the complex motor self-initiated task to the others, we decided to compare him with all the other volunteers. We found that when this task was carried out with the left hand, the activations obtained by using the Z score were significantly lower than all the other eleven volunteers in the bilateral, left and right SMA. However, when the movement was performed with the right hand, the activation observed in the left SMA was significantly higher than the others. These data support the idea that in addition to the complexity of the movement, the amount of training carried out also affects the activation of SMA [53] [54]. After learning, task difficulty decreases and unilateral brain area can accomplish the task, thus less depending on the other side of the brain [51]. In this case, it can be equally proposed that the left side of the SMA is dominant in selecting a specific action within a group of memorized tasks. This may reflect an optimization process in which the minimal circuits needed to accomplish a behavior are, with training, selected from redundant sensorimotor connections.

The limitations to this study include the small sample size typical of fMRI studies, which limits generalizability of findings. Moreover, less complex self-initiated and unpredictable sequences could be added to best demonstrate the differences in SMA activation related to the handedness.

\section{Conclusion}

In summary, we have shown bilateral SMA activation with motor tasks in normal right- and left-handed subjects. The self-initiated complex motor task induced the highest degree of SMA activation. The left SMA was significantly more activated in right- and left-handers while performing the motor tasks with the right hand. The right SMA was more activated in the left-handers when the motor tasks were executed with the left hand. These results suggest that task complexity is the most important factor influencing the degree of SMA activation and also that the laterality of SMA activation is affected by manual dominance. Given the current interest in cortical re- 
organization and neuroplasticity of the motor cortical areas and the important role played by the non-dominant hemisphere, this study provides more data regarding SMA activation and handedness in motor control, though additional experiments combining structural and functional imaging, behavior and handedness within the same group of participants are necessary to understand the determinants of lateralization.

\section{References}

[1] Kim, S.G., Ashe, J., Hendrich, K., Ellermann, J.M., Merkle, H., Ugurbil, K., et al. (1993) Functional Magnetic Resonance Imaging of Motor Cortex: Hemispheric Asymmetry and Handedness. Science, 261, 615-617. http://dx.doi.org/10.1126/science.8342027

[2] Rao, S.M., Binder, J.R., Bandettini, P.A., Hammeke, T.A., Yetkin, F.Z., Jesmanowicz, A., et al. (1993) Functional Magnetic Resonance Imaging of Complex Human Movements. Neurology, 43, 2311-2318. http://dx.doi.org/10.1212/WNL.43.11.2311

[3] Shibasaki, H., Sadato, N., Lyshkow, H., Yonekura, Y., Honda, M., Nagamine, T., et al. (1993) Both Primary Motor Cortex and Supplementary Motor Area Play an Important Role in Complex Finger Movement. Brain, 116, 1387-1398. http://dx.doi.org/10.1093/brain/116.6.1387

[4] Chung, G.H., Han, Y.M. and Kim, C.S. (2000) Functional MRI of the Supplementary Motor Area: Comparison of Motor and Sensory Tasks. Journal of Computer Assisted Tomography, 24, 521-525. http://dx.doi.org/10.1097/00004728-200007000-00002

[5] Goldberg, G. (1985) Supplementary Motor Area Structure and Function: Review and Hypothesis. Behavioral and Brain Sciences, 8, 567-616. http://dx.doi.org/10.1017/S0140525X00045167

[6] Mushiake, H., Inase, M. and Tanji, J. (1991) Neuronal Activity in the Primate Premotor, Supplementary, and Precentral Motor Cortex during Visually Guided and Internally Determined Sequential Movements. Journal of Neurophysiology, 66, 705-718.

[7] Passingham, R.E., Thaler, D.E. and Chen, Y.C. (1989) Supplementary Motor Cortex and Self-Initiated Movement. Karger, Basel.

[8] Roland, P.E., Larsen, B., Lassen, N.A. and Skinhoj, E. (1980) Supplementary Motor Area and Other Cortical Areas in Organization of Voluntary Movements in Man. Journal of Neurophysiology, 43, 118-136.

[9] Cunnington, R., Bradshaw, J.L. and Iansek, R. (1996) The Role of the Supplementary Motor Area in the Control of Voluntary Movement. Human Movement Science, 15, 627-647. http://dx.doi.org/10.1016/0167-9457(96)00018-8

[10] Dum, R.P. and Strick, P.L. (1991) The Origin of Corticospinal Projections from the Premotor Areas in the Frontal Lobe. Journal of Neuroscience, 11, 667-689.

[11] He, S.Q., Dum, R.P. and Strick, P.L. (1995) Topographic Organization of Corticospinal Projections from the Frontal Lobe: Motor Areas on the Medial Surface of the Hemisphere. Journal of Neuroscience, 15, 3284-3306.

[12] Krakauer, J. and Ghez, C. (2000) Voluntary Movement. In: Kandel, E.R., Schwartz, J.H. and Jessel, T.M., Eds., Principles of Neural Science, McGraw-Hill, New York, 756-781.

[13] Naidich, T.P., Hof, P.R., Yousry, T.A. and Yousry, I. (2001) The Motor Cortex: Anatomic Substrates of Function. Neuroimaging Clinics of North America, 11, 171-193.

[14] Briggs, G.G. and Nebes, R.D. (1975) Patterns of Hand Preference in a Student Population. Cortex, 11, $230-238$.

[15] Dalacorte, A., Portuguez, M.W., das Neves, C.M., Anes, M. and Dacosta, J.C. (2012) Functional MRI Evaluation of Supplementary Motor area Language Dominance in Right- and Left-Handed Subjects. Somatosensory and Motor Research, 29, 52-61. http://dx.doi.org/10.3109/08990220.2012.662418

[16] Strupp, J.P. (1996) Stimulate: A GUI Based fMRI Analysis Software Package. Neuroimage, 3, S607. http://dx.doi.org/10.1016/s1053-8119(96)80609-4

[17] Krings, T., Topper, R., Foltys, H., Erberich, S., Sparing, R., et al. (2000) Cortical Activation Patterns during Complex Motor Tasks in Piano Players and Control Subjects. A Functional Magnetic Resonance Imaging Study. Neuroscience Letters, 278, 189-193. http://dx.doi.org/10.1016/S0304-3940(99)00930-1

[18] Talairach, J. and Tournoux, P. (1988) Coplanar Stereotaxic Atlas of the Human Brain. Thieme, New York.

[19] Thickbroom, G.W., Byrnes, M.L., Sacco, P., Ghosh, S., Morris, I.T., et al. (2000) The Role of the Supplementary Motor Area in Externally Timed Movement: The Influence of Predictability of Movement Timing. Brain Research, 874, 233-241. http://dx.doi.org/10.1016/S0006-8993(00)02588-9

[20] Picard, N. and Strick, P.L. (1996) Motor Areas of the Medial Wall: A Review of Their Location and Functional Activation. Cerebral Cortex, 6, 342-353. http://dx.doi.org/10.1093/cercor/6.3.342

[21] Barbas, H. and Pandya, D.N. (1987) Architecture and Frontal Cortical Connections of the Premotor Cortex (Area 6) in 
the Rhesus Monkey. Journal of Comparative Neurology, 256, 211-228. http://dx.doi.org/10.1002/cne.902560203

[22] Dassonville, P., Lewis, S.M., Zhu, X.H., Ugurbil, K., Kim, S.G., et al. (1998) Effects of Movement Predictability on Cortical Motor Activation. Neuroscience Research, 32, 65-74. http://dx.doi.org/10.1016/S0168-0102(98)00064-9

[23] Hulley, S.B. and Cummings, S.R. (1988) Designing Clinical Research: An Epidemiological Approach. Williams \& Wilkins, Baltimore.

[24] Altman, D.G. (1991) Relation between Two Continuous Variables. In: Altman, D.G., Ed., Practical Statistics for Medical Research, Chapman \& Hall, London, 277-324.

[25] Hopkins, W.G. (2002) A Scale of Magnitudes for Effect Statistics: A New View of Statistics. http://www.sportsci.org/resource/stats/effectmag.html

[26] Mueller, V.A., Brass, M., Waszak, F. and Prinz, W. (2007) The Role of the preSMA and the Rostral Cingulate Zone in Internally Selected Actions. Neuroimage, 37, 1354-1361. http://dx.doi.org/10.1016/j.neuroimage.2007.06.018

[27] Witt, S.T., Laird, A.R. and Meyerand, M.E. (2008) Functional Neuroimaging Correlates of Finger-Tapping Task Variations: An ALE Meta Analysis. Neuroimage, 42, 343-356. http://dx.doi.org/10.1016/j.neuroimage.2008.04.025

[28] Tanji, J. and Shima, K. (1994) Role for Supplementary Motor Area Cells in Planning Several Movements Ahead. Nature, 371, 413-416. http://dx.doi.org/10.1038/371413a0

[29] Shima, K. and Tanji, J. (1998) Role for Cingulate Motor Area Cells in Voluntary Movement Selection Based on Reward. Science, 282, 1335-1338. http://dx.doi.org/10.1126/science.282.5392.1335

[30] Hikosaka, O., Sakai, K., Miyauchi, S., Takino, R., Sasaki, Y., et al. (1996) Activation of Human Pre-Supplementary Motor Area in Learning of Sequential Procedures: A Functional MRI Study. Journal of Neurophysiology, 76, 617-621.

[31] Humberstone, M., Sawle, G.V., Clare, S., Hykin, J., Coxon, R., et al. (1997) Functional Magnetic Resonance Imaging of Single Motor Events Reveals Human Presupplementary Motor Area. Annals of Neurology, 42, 632-637. http://dx.doi.org/10.1002/ana.410420414

[32] Lee, K.M., Chang, K.H. and Roh, J.K. (1999) Subregions within the Supplementary Motor Area Activated at Different Stages of Movement Preparation and Execution. Neuroimage, 9, 117-123. http://dx.doi.org/10.1006/nimg.1998.0393

[33] Richter, W., Andersen, P.M., Georgopoulos, A.P. and Kim, S.G. (1997) Sequential Activity in Human Motor Areas during a Delayed Cued Finger Movement Task Studied by Time-Resolved fMRI. Neuroreport, 8, 1257-1261. http://dx.doi.org/10.1097/00001756-199703240-00040

[34] Schubert, T., von Cramon, D.Y., Niendorf, T., Pollmann, S. and Bublak, P. (1998) Cortical Areas and the Control of Self-Determined Finger Movements: An fMRI Study. Neuroreport, 9, 3171-3176. http://dx.doi.org/10.1097/00001756-199810050-00009

[35] Van Oostende, S., Van Hecke, P., Sunaert, S., Nuttin, B. and Marchal, G. (1997) FMRI Studies of the Supplementary Motor Area and the Premotor Cortex. Neuroimage, 6, 181-190. http://dx.doi.org/10.1006/nimg.1997.0287

[36] Wexler, B.E., Fulbright, R.K., Lacadie, C.M., Skudlarski, P., Kelz, M.B., et al. (1997) An fMRI Study of the Human Cortical Motor System Response to Increasing Functional Demands. Magnetic Resonance Imaging, 15, 385-396. http://dx.doi.org/10.1016/S0730-725X(96)00232-9

[37] Wildgruber, D., Erb, M., Klose, U. and Grodd, W. (1997) Sequential Activation of Supplementary Motor Area and Primary Motor Cortex during Self-Paced Finger Movement in Human Evaluated by Functional MRI. Neuroscience Letters, 227, 161-164. http://dx.doi.org/10.1016/S0304-3940(97)00329-7

[38] Freund, H.J. (1996) Functional Organization of the Human Supplementary Motor Area and Dorsolateral Premotor Cortex. Advances in Neurology, 70, 263-269.

[39] Luders, H.O. (1996) The Supplementary Sensorimotor Area: An Overview. Advances in Neurology, 70, 1-16.

[40] Scholz, V.H., Flaherty, A.W., Kraft, E., Keltner, J.R., Kwong, K.K., et al. (2000) Laterality, Somatotopy and Reproducibility of the Basal Ganglia and Motor Cortex during Motor Tasks. Brain Research, 879, 204-215. http://dx.doi.org/10.1016/S0006-8993(00)02749-9

[41] Krainik, A., Duffau, H., Capelle, L., Cornu, P., Boch, A.L., et al. (2004) Role of the Healthy Hemisphere in Recovery after Resection of the Supplementary Motor Area. Neurology, 62, 1323-1332. http://dx.doi.org/10.1212/01.WNL.0000120547.83482.B1

[42] Halsey Jr., J.H., Blauenstein, U.W., Wilson, E.M. and Wills, E.H. (1979) Regional Cerebral Blood Flow Comparison of Right and Left Hand Movement. Neurology, 29, 21-28. http://dx.doi.org/10.1212/WNL.29.1.21

[43] Toyokura, M., Muro, I., Komiya, T. and Obara, M. (1999) Relation of Bimanual Coordination to Activation in the Sensorimotor Cortex and Supplementary Motor Area: Analysis Using Functional Magnetic Resonance Imaging. Brain Research Bulletin, 48, 211-217. http://dx.doi.org/10.1016/S0361-9230(98)00165-8

[44] Schmidt, S.L., Oliveira, R.M., Krahe, T.E. and Filgueiras, C.C. (2000) The Effects of Hand Preference and Gender on 
Finger Tapping Performance Asymmetry by the Use of an Infra-Red Light Measurement Device. Neuropsychologia, 38, 529-534. http://dx.doi.org/10.1016/S0028-3932(99)00120-7

[45] Herve, P.Y., Mazoyer, B., Crivello, F., Perchey, G. and Tzourio-Mazoyer, N. (2005) Finger Tapping, Handedness and Grey Matter Amount in the Rolando’s Genu Area. Neuroimage, 25, 1133-1145. http://dx.doi.org/10.1016/j.neuroimage.2004.12.062

[46] Lutz, K., Koeneke, S., Wustenberg, T. and Jancke, L. (2005) Asymmetry of Cortical Activation during Maximum and Convenient Tapping Speed. Neuroscience Letters, 373, 61-66. http://dx.doi.org/10.1016/j.neulet.2004.09.058

[47] Mattay, V.S., Callicott, J.H., Bertolino, A., Santha, A.K., Van Horn, J.D., et al. (1998) Hemispheric Control of Motor Function: A Whole Brain Echo Planar fMRI Study. Psychiatry Research, 83, 7-22. http://dx.doi.org/10.1016/S0925-4927(98)00023-7

[48] Fried, I., Katz, A., McCarthy, G., Sass, K.J., Williamson, P., et al. (1991) Functional Organization of Human Supplementary Motor Cortex Studied by Electrical Stimulation. Journal of Neuroscience, 11, 3656-3666.

[49] Kawashima, R., Roland, P.E. and O’Sullivan, B.T. (1994) Activity in the Human Primary Motor Cortex Related to Ipsilateral Hand Movements. Brain Research, 663, 251-256. http://dx.doi.org/10.1016/0006-8993(94)91270-X

[50] Roland, P.E. and Zilles, K. (1996) Functions and Structures of the Motor Cortices in Humans. Current Opinion in Neurobiology, 6, 773-781. http://dx.doi.org/10.1016/S0959-4388(96)80027-4

[51] Zhu, Y., Dong, Z., Weng, X. and Chen, Y. (2005) Functional Brain Laterality for Sequential Movements: Impact of Transient Practice. Chinese Science Bulletin, 50, 235-239. http://dx.doi.org/10.1360/982004-325

[52] Verstynen, T., Diedrichsen, J., Albert, N., Aparicio, P. and Ivry, R.B. (2005) Ipsilateral Motor Cortex Activity during Unimanual Hand Movements Relates to Task Complexity. Journal of Neurophysiology, 93, 1209-1222. http://dx.doi.org/10.1152/jn.00720.2004

[53] Erdler, M., Windischberger, C., Lanzenberger, R., Edward, V., Gartus, A., et al. (2001) Dissociation of Supplementary Motor Area and Primary Motor Cortex in Human Subjects When Comparing Index and Little Finger Movements with Functional Magnetic Resonance Imaging. Neuroscience Letters, 313, 5-8. http://dx.doi.org/10.1016/S0304-3940(01)02167-X

[54] Jancke, L., Shah, N.J. and Peters, M. (2000) Cortical Activations in Primary and Secondary Motor Areas for Complex Bimanual Movements in Professional Pianists. Cognitive Brain Research, 10, 177-183. http://dx.doi.org/10.1016/S0926-6410(00)00028-8 